\title{
3D Magnetic Analysis of Permanent Magnets in Spherical Configuration
}

\author{
Yusuf Oner ${ }^{\dagger}$ and Selami Kesler*
}

\begin{abstract}
The present study aims to increase the amount of surface flux by changing the magnetic directions of a spherical magnet $(\mathrm{NdFeB})$ consisting of four poles. For this purpose, the magnetic directions of quartile spherical slices constituting the spherical magnet are manipulated and their threedimensional analyses are carried out by using finite-element method via Maxwell environment. The analysis of the magnetic quartile spheres with four different magnetic directions are compared to the each other, and then the quartile sphere with the best surface flux distribution is suggested for rotor structure. It is clear emphasized that the induced torque of the spherical motor, in which such a rotor is used, will be improved as well.
\end{abstract}

Keywords: Spherical magnet, Spherical motor, 3D magnetic analysis

\section{Introduction}

Permanent magnets have unique abilities over conventional electromagnets: they can continuously generate magnetic flux without consumption of input energy and no power for cooling. Especially, the neodymium-iron-boron $(\mathrm{NdFeB})$ magnet developed in 1980s has enlarged the application of permanent magnets in the electrical industry [1-2]. The Halbach magnet array was proposed by Halbach in 1980 for undulatory or electron wiggler [3]. If there is a magnet for which the magnetization direction rotates continuously, magnetic flux is constructed via superposition on one side of the magnet while the flux is destructed on the other side. Since continuously rotating magnetization is difficult to manufacture, the ideal array is approximated with a segmented magnet [4]. Bojan Stumberger et al. point out the comparison of torque capability of the three-phase permanent magnet synchronous motors with different permanent magnet arrangements [5]. Moon G. Lee and Dae-Gab Gweon emphasized the multi-segmented trapezoidal magnet array for a high-precision positioning system [6]. More and more analytical approaches were proposed by many authors for calculating the magnetic field created by arc-shaped permanent magnets whose polarizations can be radial [7-9]

The design of the spherical magnet can also be the permanent magnet array. Actually, the spherical permanent magnet array (SPMA) is generally used in spherical motors. Spherical motors can be described as motors that have movement capability that can be possessed by one or more than one motor. Unlike other electric motors, their movement capability is not mono-axial; they can move along different axes. Due to this characteristic, they are

\footnotetext{
$\dagger \quad$ Corresponding Author: Dept. of Electrical and Electronic Engineering, Pamukkale University, Turkey. (yoner@pau.edu.tr)

* Dept. of Electrical and Electronic Engineering, Pamukkale University, Turkey. (skesler@pau.edu.tr)

Received: August 7, 2014; Accepted: July 19, 2015
}

chosen in industrial for different purposed applications. However, they have not achieved commercial success yet because they do not have a sound structure, and they have a complex electric and magnetic design.

A lot of research about spherical motors have been going on for several decades. There are many different structured spherical motors resembling induction motors [10, 11], direct-current motors [12-14], stepper motors [15], variable reluctance motors $[16,17]$ and ultrasonic motors $[18,19]$. Three dimensional nature of the electromagnetic field distribution in the foregoing spherical motors make their electromagnetic and dynamic behavior difficult to analyze, and this has been a significant obstacle to their design optimization and servo application. As a result, the potential benefits of employing spherical motors have not been realized. Therefore, most of these motor forms remain un-commercialized. In recent past, new formulas of the spherical actuators moving in two-free axes are analyzed and approved with the help of experiences [20,21].

And the features of rotors used in these designs are very different from each other. The directions of the magnets in the form of pieces used in a spherical motor to be designed should be selected in such a way that the surfaces at flux values can evenly be distributed [22-24]. Hongfeng Li and et al carried out the spherical harmonic analysis of a halbach array permanent magnet spherical motor [25]. Design and analysis of a variable arc permanent magnet array for the spherical motor were reported by Changliang Xia and et al. [26]. Hyun-Jong Park and et al introduced a several studies about performance increasing of permanent magnet spherical motor. [27-29]. Bin Li and et al implemented a spherical motor design by using spherical harmonics [30]. Zhe Qian and et al studied on analysis of permanent magnet spherical motor with cylindrical poles and reported effects of that pole structure [31]. Hyung-Woo Lee et al focused on calculation of the produced torque in permanent magnet spherical motors [32]. Guo Jinjun et al investigated the effect of pole layouts 
of permanent magnets and electromagnets on inclination torque of a multi degrees of freedom spherical motor [33].

As known, the directions of permanent $\mathrm{NdFeB}$ magnets are determined during production, so they possess predetermined magnetic characteristics. So, in this study, the permanent magnet spherical rotor structures are investigated. For this purpose, magnetic analyses of this rotor type are made via simulations by changing magnetic directions of the quartile spherical slices forming the rotor to find out the increase in the surface flux values.

For each rotor structure, analysis is carried out for the permanent magnet material with the same features. In this way the possibility of making comparison is created.

\section{Rotor Structure and Magnetic Directions}

The spherical magnet introduced in this study consists of combining four-quartile spheres. Every quartile includes the same $\mathrm{NdFeB} 35$ material as a single piece. The permanence value of the $\mathrm{NdFeB} 35$ permanent magnet is $\mathrm{Br}=1,234 \mathrm{~T}$, and its relative permeability is $\mu \mathrm{r}=1,15$ [34]. Fig. 1. shows the spherical rotor structure designed with four pieces of permanent magnets, and the B-H curve for the spherical magnet can be seen in Fig. 2 .

The magnetic directions of the four pieces of quartile spheres forming the spherical magnet are changed in four different ways, and their magnetic analyses are conducted for each changing.

Every variation of the magnetic-field directions results in a change of flux directions inside and on the surface of the spherical magnet, and in this way, the magnetic direction yielding the highest flux value on the surface of the sphere is achieved for the rotor using in the application.

$\mathrm{Br}(1,234 \mathrm{~T})$ is the value computed in the conditions of a closed loop (magnetic core) magnetic circuit [34, 36]. This value was calculated to be around $0.6 \mathrm{~T}$ on the surface of the magnet (without magnetic core).

In numerical calculations made for the geometry of the spherical motor designed by Howe et al., the flux values on the surface of the spherical rotor were calculated with legendry polynomial. Since the same structure is used, the same calculations are directly used here

\section{3D Magnetic Analyses}

3D magnetic analyses are carried out for 4-different modeled spherical permanent magnets. The magnetic dimension of Model-1 is adjusted in such a way as to pass through the middle of the quartile magnetic slice and at $\alpha=45^{\circ}$. Two opposite poles have $\mathrm{N}$ magnetic direction, and the other two opposite poles have $\mathrm{S}$ magnetic direction.spherical coordinate system

Model-2 is adjusted in such a way as to have a magnetic direction of $\alpha=90^{\circ}$; the magnetic dimension of Model-3 is

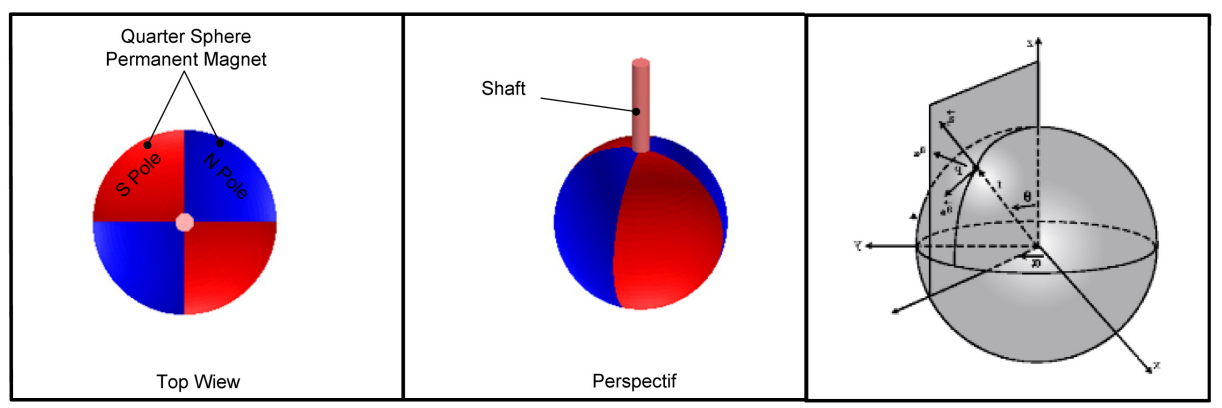

Fig. 1. Permanent magnet rotor in spherical structure and Ansoft Maxwell software has been used for the proposed design and simulations [35].

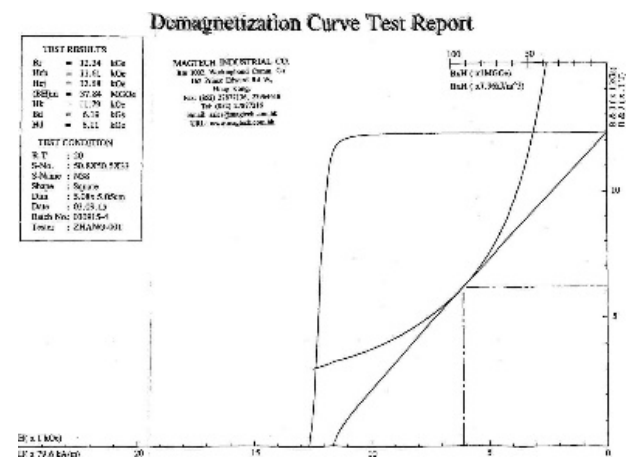

\begin{tabular}{c|c}
\hline \multicolumn{2}{c}{ Material Specifications } \\
\hline Rel. Permittivity & 1 \\
\hline Rel. Permeability & 1.099778 \\
\hline Conductivity & $6.25 \times 10^{5}$ \\
\hline Thermal Expansion & 1 \\
\hline Mag. Coercivity & $-8.9 \times 10^{5}$ \\
\hline Mag. Retentivity & 1.23 \\
\hline Magnetization & 978802.901 \\
\hline
\end{tabular}

Fig. 2. B-H curve and material specifications of the permanent magnet supported by the manufacturer. 


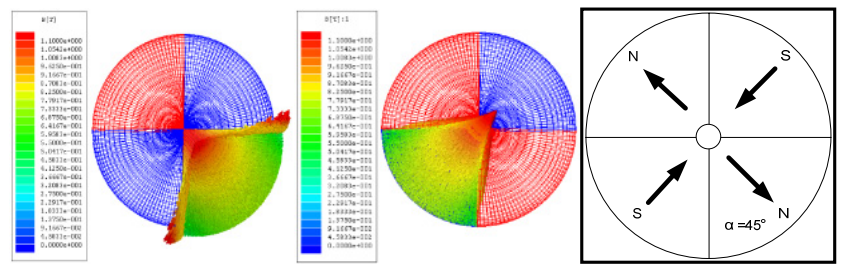

(a)

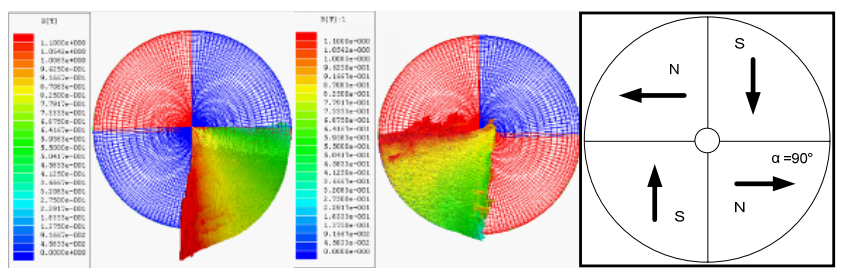

(c)

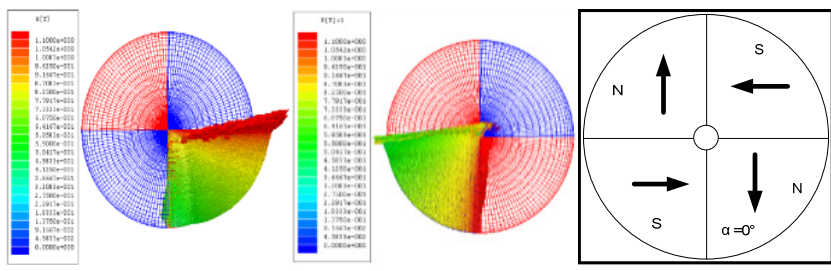

(b)
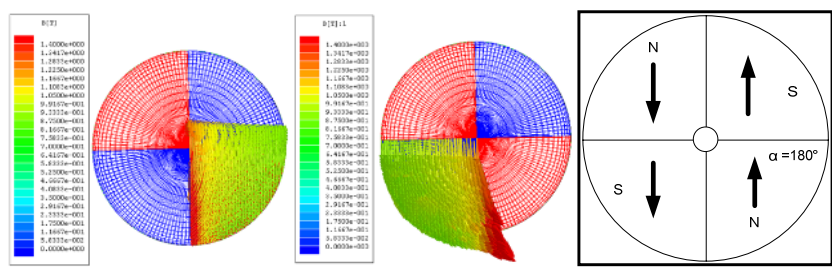

(d)

Fig. 3. The vector images of the flux intensity of the designed 4-different model spheres are: (a) Model-1 magnetic direction of the magnet $\alpha=45^{\circ}$; (b) Model-2 magnetic direction of the magnet $\alpha=90^{\circ}$; (c) Model-3 magnetic direction of the magnet $\alpha=0^{\circ}$; (d) Model-4 magnetic direction of the magnet $\alpha=180^{\circ}$.
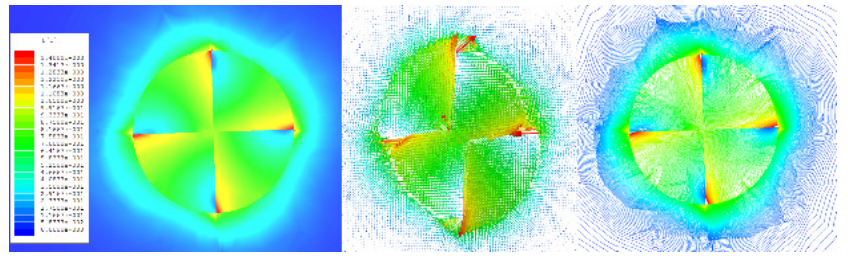

(a)
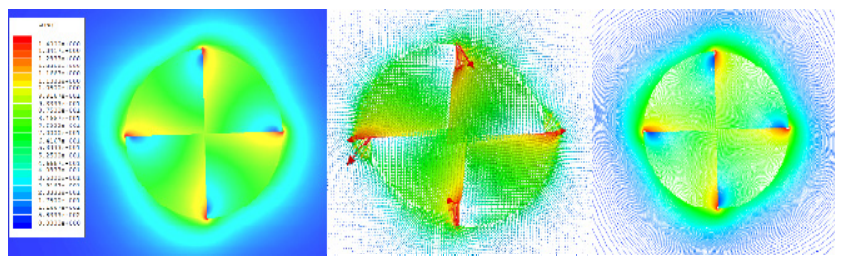

(c)
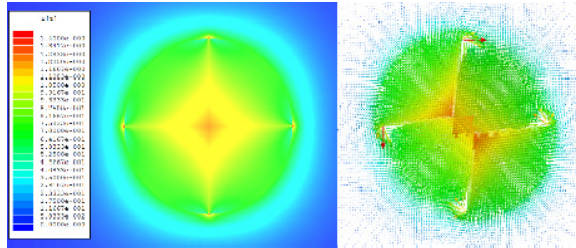

(b)
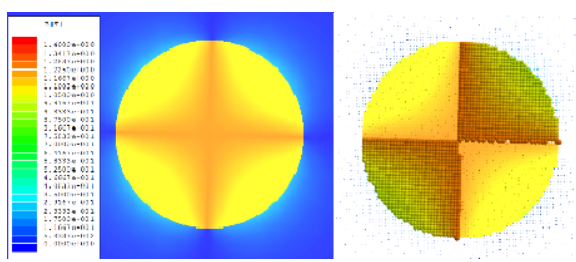

(d)

Fig. 4. (a) Model-1; (b) Model-2; (c) Model-3; (d) Model-4, magnetic flux density on the central section, vector image of the flux and distribution of the flux lines inside the sphere and on the surface of it, respectively.

adjusted to be $\alpha=0^{\circ}$; and the magnetic direction of Model4 is adjusted to be $\alpha=180^{\circ}$. In Fig. 3. the vector images of the flux densities on the surface of the sphere can be seen. While one pole is directed outside, the other one is directed from outside to inside.

At the end of the analyses carried out, when a section is taken from the center of the spherical magnet and then its magnetic flux lines are examined, many different magnetic distributions will be seen, as in Fig. 4. The purpose of the present study is to create a high magnetic flux density and even flux distribution on the surface of the sphere. In Fig. 4. the magnetic flux distribution occurring on the central area is shown in the form of vector and power lines. By comparing these different analyses, information of the strength of the flux on the surface of the magnet can be obtained.

In line with the architecture of the spherical motor, the external surface of the spherical magnet that can be used should be one pole, and the internal side should be one pole. Therefore, the best flux distribution can be seen in Model-1. Among the other models, Model-1 can be selected for spherical rotor application as it yields the highest level of flux and the smoothest flux. In Model-4, there is no good flux distribution on the surface in spite of high flux density. Therefore, structure of Model-1 which has low harmonic order and good flux distribution can be employed as a rotor for the spherical motors.

Magnetic poles occurring on the surfaces of the magnetic spheres depending on their directions can clearly be seen in Fig. 5. Here, the sections marked with red represent the $\mathrm{N}$ pole, and the sections marked with blue represent the $\mathrm{S}$ pole. When we look at the Fig. 5. attached to Model-1, we can see the inner edge become the $\mathrm{N}$ pole and the external surface becomes its opposite the $\mathrm{S}$ pole. In 

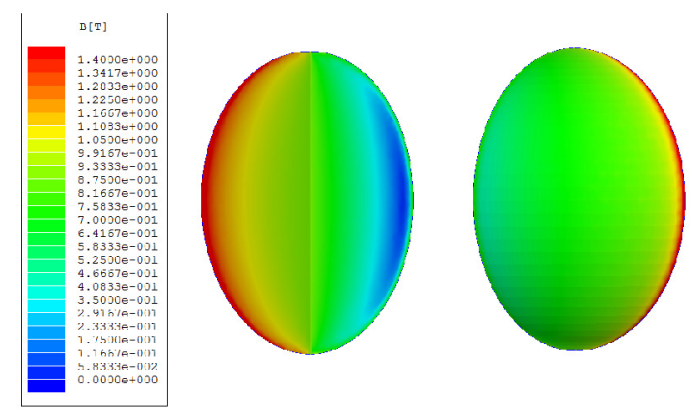

(a)
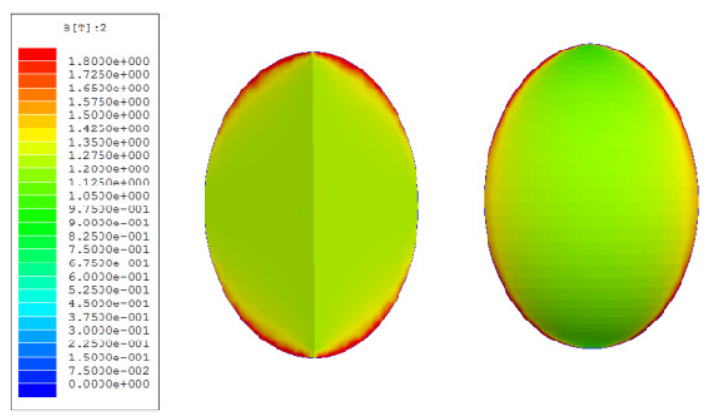

(c)
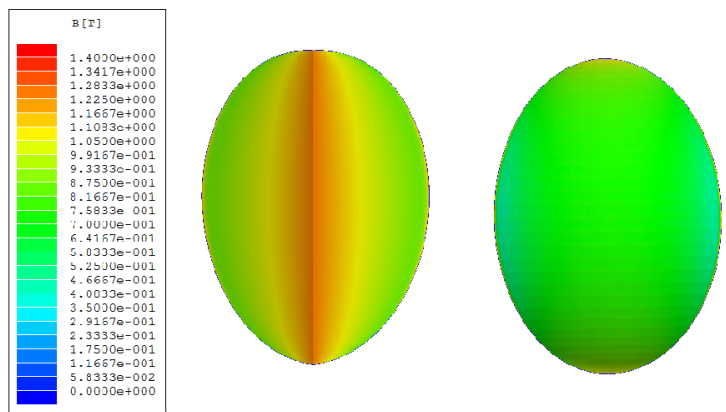

(b)
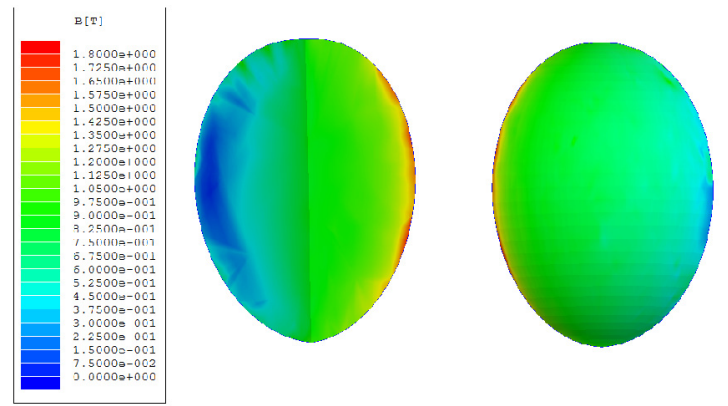

(d)

Fig. 5. Polarization of the quartile spherical slice in (a) Model-1; (b) Model-2; (c) Model-3; (d) Model-4

this way, the external surface of the quartile sphere slice creates a pole extending from the center towards the outside. This is very suitable for the spherical motor architecture and completes movement of the spherical rotor.

\section{Calculation of Magnetic Flux Distributions}

The magnetic field region generated by the permanent magnet spherical rotor lays on the basis of distribution information, design optimization and the creation of the proper model for the spherical motor [20]. Given the fact that magnetic flux lines extend on the soft iron stator without leakage, the magnetic field region can be divided into two sub-areas. Air gap having $\mu_{0}$ magnetic permeability and coil region which is the permanent magnet surface having $\mu_{0} \mu_{\mathrm{r}}$ magnetic permeability. Therefore,

$$
B=\left\{\begin{array}{l}
\mu_{0} \cdot H \\
\mu_{0} \cdot \mu_{r} \cdot H+\mu_{0} \cdot M
\end{array}\right.
$$

Here $\mu_{r}$ is the magnet relative permeability and $\mathbf{M}$ is the magnetization vector based on residue magnetization. The $\mu_{r}$ value of a permanent magnet having linear demagnetization characteristics is constant and there is a $\mathrm{M}=B_{r} / \mu_{0}$ relationship between the magnetization vector, $\mathbf{M}$ and the permanent magnetism, $B_{r}$.

The radial constituent of the magnetization vector can be expressed as follows by extending in the form of $M_{r}$ spherical harmonics:

$$
M_{r}=\sum_{l=2,4}^{\infty} \sum_{m=1,3}^{l} M_{l m} P_{l}^{m}(\cos \theta) \cdot \sin \alpha
$$

where, $P_{l}^{m}(),$.$m is associated with ordinary l$. degree of Legendre polynomial and $M_{l m}$ can be expressed as follows [20].

$$
\begin{gathered}
M_{l m}=\frac{4(2 l+1)(l-m) !}{\pi m(l+m) !} M_{c} \int_{0}^{l} P_{l}^{m}(x) x d x \\
M_{c}=B_{r} / \mu_{0} \sqrt{2}
\end{gathered}
$$

By considering the radial constituent boundary conditions of the distribution, the flux density on the spherical permanent magnet surface can be calculated by Eq. (5).

$$
B_{l r}=\sum_{l=2,4}^{\infty} \sum_{m=1,3}^{l} C_{l m}(l+1) r^{-(l+2)} \cdot P_{l}^{m}(\cos \theta) \sin \alpha
$$

where,

$$
C_{l m}=\mu_{0} R_{m}{ }^{l+2} M_{l m} /\left[\left(1+l\left(1+\mu_{r}\right)\right]\right.
$$

And, $R_{m}$ is the radius of the spherical rotor. 


\section{Results and Discussion}

The magnetic flux densities of the rotor are calculated by Eq. (5) for the position of $R_{m}=30 \mathrm{~mm}, r=0.031 \mathrm{~m}$ and $\theta=0^{\circ}$ for the gap between $\alpha=-\pi$ and $+\pi$. For the radial constituents of the magnetic flux densities, a comparison of the results between calculated by analytical method and obtained by finite elements method is given in Fig. 6, for designed model-1.

In order to measure the fluxes on the spherical magnet surfaces, the mechanism illustrated in Fig. 7 is established, and surface fluxes of the spherical magnet can be depicted graphically. The spherical magnet is mounted to a step motor shaft and rotated step by step by the computer-aided program. At each step, it waits for 2 seconds, and then it rotates one more step. During the waiting time, the magnetic flux value on the surface of the spherical magnet is measured by the sensor of gauss meter and then all of the measured data are transferred into the computer. The obtained data are tabulated by an Excel page. By using the stored data, the flux densities on the surface of the spherical magnet are plotted as shown in Fig. 8. The Gauss Meter being used for the measurements has a Tesla Unit option.

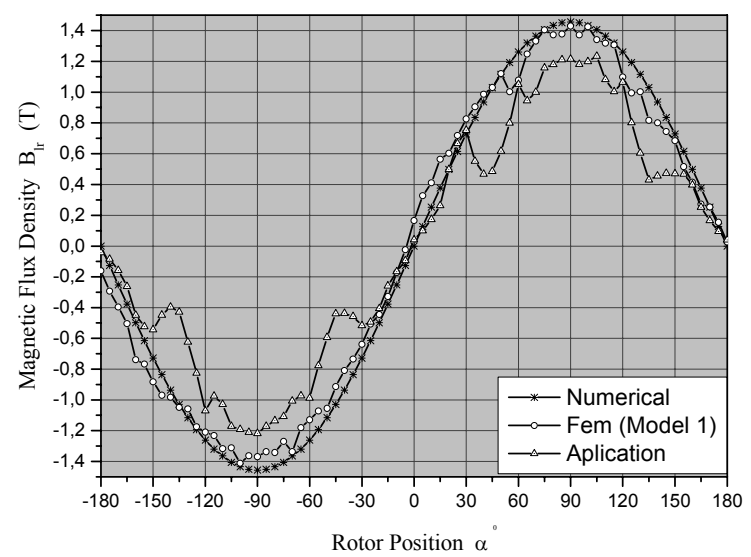

Fig. 6. Comparison of the results for the radial constituents of magnetic flux densities for the $\theta=0^{\circ}$ position of the rotor for the gap between $\alpha=-\pi$ and $+\pi$ calculated by analytical method and finite elements method.

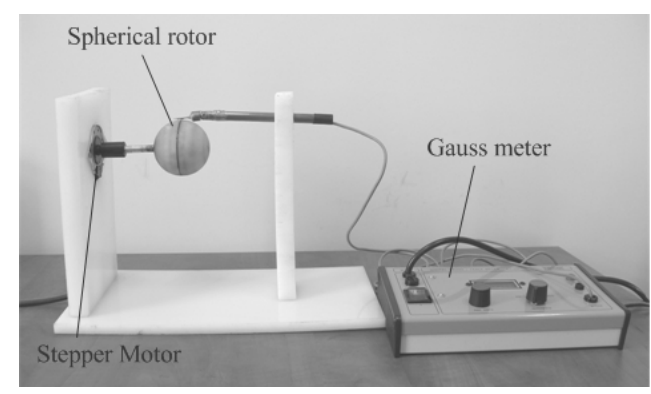

Fig. 7. Measurement system for flux density of the spherical magnet

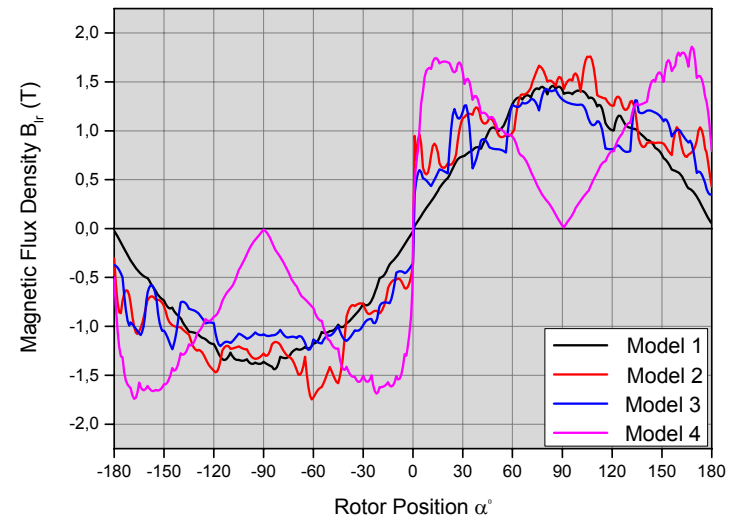

Fig. 8. At $\theta=0^{\circ}$ position of the rotor, the comparison of the radial constituents of the magnetic flux densities for the gap between $\alpha=-\pi$ and $+\pi$ Model 1-2-3-4.

Simulation results obtained by changing of magnetic field vectors of the magnets for Model-1 among the studied models complies with the results obtained by calculation with a numerical method as shown in Fig. 6, where surface flux density of the magnet is calculated by a numerical method, and FEM, and measured by an experiment.

From Fig. 8, it can be seen that the highest flux value on the surface is obtained from Model-1. On the other models, the surface flux values are lower than Model-1 and the fluxes on the surfaces are not homogeneous. Comparisons of calculations and measurements on the spherical magnet surfaces in terms of magnetic flux density are given in Fig. 6 and Fig. 8. Examining Model-2 and Model-3, the flux values are high, but have too harmonic components. For Model-4, the magnetic flux does not make a sine wave at all. But, the results obtained from Model 1 meet the expectations for a good structure of spherical rotor by permanent magnets.

\section{Conclusion}

In this study, four-different manipulated spherical magnet are designed and their three-dimensional analyses are realized by using finite-element method via Maxwell environment. The analysis of the magnetic quartile spheres with four different magnetic directions are compared to the each other, and then the quartile sphere with the best surface flux distribution is suggested for rotor structure.

Due to its high and smooth surface flux, which is very important to the architecture of the spherical motor, Model1 can be used for the spherical motor to get desired torque characteristic. By manipulating different magnet geometries or making different spherical magnet designs, the surface flux values can be improved. In future studies, by using magnets with four different magnetic directions, more available spherical structure can be formed, and more improved flux density on the surface can be achieved, as well. 


\section{References}

[1] J.M.D. Coey., "Permanent Magnet Applications" Journal of Magnetism and Magnetic Materials, Vol. 248 pp. 441-456, 2002.

[2] Jae Seok Choi, Jeonghoon Yoo, "Optimal Design Method for Magnetization Direction of a Permanent Magnet Array" Journal of Magnetism and Magnetic Materials, Vol. 322 pp. 2145-2151, 2010.

[3] Moon G. Lee., Sung Q. Lee., Dae-Gab Gweon., "Analysis of Halbach Magnet Array and its Application to Linear Motor" Mechatronics, vol. 14, pp. 115-128, 2004.

[4] Ofori-Tenkorang J., Lang J. H., "A Comparative Analysis of Torque Production in Halbach and Conventional Surface-Mounted Permanent Magnet Synchronous motors" Conference Proceedings IEEE IAS 30th Annual meeting, pp. 657-663, 8-12 October 1995.

[5] Bojan Stumberger, Gorazd Stumberger, Miralem Hadziselimovic, Anton Hamler, Viktor Gorican, Marko Jesenik, Mladen Trlep., "Comparison of Torque Capability of Three-Phase Permanent Magnet Synchrounos Motors with Different Permanent Magnet Arrangement" Journal of Magnetism and Magnetic Materials, vol. 316, pp. 261-264, 2007.

[6] Moon G. Lee, Dae-Gab Gweon., "Optimal Design of a Double-Sided Linear Motor with a MultiSegmented Trapezoidal Magnet Array for a High Precision Positioning System" Journal of Magnetism and Magnetic Materials, vol. 281, pp. 336-346, 2004.

[7] Babic, S. I. and C. Akyel, "Improvement in the analytical calculation of the magnetic field produced by permanent magnet rings," Progress In Electromagnetics Research C, vol. 5, pp. 71-82, 2008.

[8] Ravaud, R., G. Lemarquand, V. Lemarquand, and C. Depollier,"The three exact components of the magnetic field created by a radially magnetized tile permanent magnet," Progress In Electromagnetics Research, PIER vol. 88, pp. 307-319, 2008.

[9] Ravaud, R., G. Lemarquand, V. Lemarquand, and C. Depollier, "Discussion about the analytical calculation of the magnetic field created by permanent magnets," Progress In Electromagnetics Research B, vol. 11, pp. 281-297, 2009.

[10] Davey., K., Vachtsevanos., G.J. ve Power., R. “An Analysis of Fields and Torques in Spherical Induction Motors" IEEE Transactions on Magnetics, vol. 23, pp. 273-281, 1987.

[11] Foggia., A., Oliver., E., Chappuis., F. "New Three Degree of Freedom Electromagnetic Actuator", Conference Record IAS Anual Meeting 35, New York, 1988.

[12] Hollis., R.L., Ralph L., "Magnetically Levitated Fine Motion Robot Wrist with Programmable Compliance" EP Pat. EP0294760 A1 1989.
[13] Kaneko., K., Yamada. I, nad Itao. K. "A Spherical DC Servo Motor with Three Degrees of Freedom" Transactions of the ASME Journal of Dynamic System Measurement and Control, vol. 111, pp. 398-402, 1989.

[14] C.K. Lim, L. Yan, I.M. Chen, et al., Mechanical design \& numerical electromagnetic analysis of a DC spherical actuator, Conference on Robotics, Automation and Mechatronics, Proceedings of the IEEE, pp. 536-541, 2004.

[15] Lee., K.M. Kwan, C., "Design Concept Development of A Spherical Stepper for Robotic Applications" IEEE Transactiona on Robotics and Automation, vol. 7, pp. 175-181, 1991.

[16] Roth., R. Lee., K.M., "Design Optimisation of A Three Degrees of Freeedom Variable Reluctance Spherical Wrist Motor" Transactions of the ASME Journal of Engineering for Industry, vol. 117, pp. 378-388, 1995.

[17] Zhou., Z. Lee., K.M., "Real Time Motion Control of Multi Degree of Freedom Variable Reluctance Spherical Motor", Proceedings of the 1996 IEEE International Conference on Robotics and Automation, pp. 2859-2864, Minneopolis-Minnesota, 1996.

[18] C. Zhao, Z. Li, W. Huang, Optimal design of the stator of a three-DOF ultrasonic motor, Sensors Actuators A, vol. 121, pp. 494-499, 2005.

[19] T. Shigeki, Z. Guoqiang, M. Osamu, Development of new generation spherical ultrasonic motor, International Conference on Robotics and Automation, Proceedings of the IEEE, pp. 2871-2876, 1996.

[20] Wang., J., Jewell., G., Howe., D. “Analysis, Design and Control of Novel Spherical Permanent Magnet Actuator" IEE Proceedings on Electrical Power Applications. vol. 145, pp. 61-71, 1998.

[21] Wang., J., Jewell., G., Howe., D. "Modeling of a Novel Spherical Permanent Magnet Actuator", Proceeding of IEEE International Conference on Robotics and Automation pp. 1120-1125, New Mexico, 1997.

[22] S. J. Lee and D.C. Jiles "Modeling and simulation of a permanent magnet array in elliptical configurations" MSM 2000 Technical Proceedings of the 2000 International Conference on Modeling and Simulation of Microsystems, pp. 668-671, 2000.

[23] Oner Y., "A Permanent Magnet Spherical Rotor Design and Three Dimensional Static Magnetic Analysis", Sensors and Actuators A, vol. 137, pp. 200-208, 2007.

[24] Xu Yanliang, Feng Kaijie, "Analysis on Toothless Permanent Magnet Machine with Halbach Array" IPEMC 2006.

[25] Hongfeng Li, Changliang Xia and Tingna Shi, "Spherical Harmonic Analysis of a Novel Halbach Array PM Spherical Motor" Proceeding of IEEE International Conference on Robotics and Biomimetics, pp. 2085-2089, Sanya, China, 2007. 
[26] Changliang Xia, Jianguo Xin, Hongfeng Li and Tingna Shi, "Design and Analysis of a Variable Arc Permanent Magnet Array for Spherical Motor" IEEE Transactions on Magnetics, vol. 49, no. 4, pp. 14701478, 2013.

[27] Hyun-Jong Park, Ho-Joon Lee, Su-Yeon Cho, HanWoong Ahn, Ki-Doek Lee, Chan-Yeop Park, SungHong Won and Ju Lee, "A Performance Study on a Permanent Magnet Spherical Motor" IEEE Transactions on Magnetics, vol. 49, no. 5, pp. 2307-2310, 2013.

[28] Hyun-Jong Park, Su-Yeon Cho, Han-Woong Ahn, Ho-Joon Lee, Sung-Hong Won and Ju Lee, “A Study of Advanced Spherical Motor for Improvement of Multi-DOF Motion" Journal of Electrical Engineering \& Technology, vol. 7, no. 6, pp. 926-931, 2012.

[29] Ho-Joon Lee, Hyun-Jong Park, Sung-Hong Won, Gwang-Hyun Ryu, and Ju Lee, "Improvements of Performance of Multi-DOF Spherical Motor by Doubled Air-gap Feature" Journal of Electrical Engineering \& Technology, vol. 8, no. 1, pp. 90-96, 2013.

[30] Bin Li, Rujian Yu, Hua Li, and Guidan Li, "Design Considerations of a Permanent Magnetic Spherical Motor Using Spherical Harmonics", IEEE Transactions on Magnetics, vol. 50, no. 8, 2014.

[31] Zhe Qian, Qunjing Wang, Guoli Li, Xiwen Guo, Cungang Hu, and Hui Yan, "Design and Analysis of Permanent Magnetic Spherical Motor with Cylindrical Poles" International Conference on Electrical Machines and Systems, pp. 644-649, Busan, Korea, 2013.

[32] Hyung-Woo Lee, Dong-Woo Kang, and Ju Lee "Torque Calculation Method of a Permanent Magnet Spherical Motor" Journal of Electrical Engineering \& Technology, vol. 5, no. 3, pp. 431-434, 2010.

[33] Guo Jinjun, Dong-Hoon Kim, Hungsun Son, "Effects of Magnetic Pole Design on Orientation Torque for a Spherical Motor" IEEE/ASME Transactions on Mechatronics, vol. 18, no. 4, pp. 1420-1425, 2013.

[34] Oner, Y., "Sabit Miknatıslı Küresel Motorun Bilgisayar Destekli Üç Boyutlu Manyetik Analizi, Tasarımı ve Uygulaması", Ph.D Thesis, Gazi University, Institıte of Technology, July, 2004.

[35] Ansoft: Maxwell 3D, Getting started: a 3D Magnetic Force Problem, 2000.

[36] Changliang Xia, Hongfeng Li, and Tingna Shi, "3D Magnetic Field and Torque Analysis of a Novel Halbach Array Permanent-Magnet Spherical Motor" IEEE Transactions on Magnetics, vol. 44, no. 8, 2008.

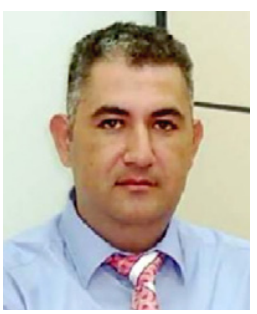

Yusuf Oner $\mathrm{He}$ received the B.Sc. degree from the Gazi University, Turkey in 1993, the M.Sc. degree from the University of Gazi, Turkey in 1998, all in electrical education. He received the Ph.D. degree in electrical education from the Gazi University, Turkey in 2004. In 1999, he joined the Gazi University, and he was appointed as a Research and Teaching Assistant from 1999 to 2004. He joined the Department of Electrical and Electronic Engineering, Pamukkale University, Denizli, Turkey, in 2007 as a Associate prof. Dr. His research interests include 3D magnetic analysis, magnetic bearing, electrical machine design and analysis, spherical motor.

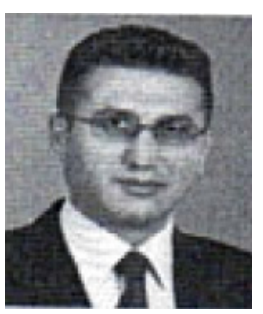

Selami Kesler $\mathrm{He}$ received the BSc, MSc and PhD Degrees from Karadeniz Technical University, Turkey in 1991, in 1998 and in 2006 respectively all in Electrical Engineering. He joined Karadeniz Technical University in 1992 and he worked as a lecturer until 2004. He joined the Department of Electrical Education, Pamukkale University, Denizli, Turkey, in 2004 as a lecturer. He joined the Department of Electrical and Electronic Engineering, Pamukkale University, Denizli, Turkey, in 2007 as a assistant professor. His fields of interest are power electronics, $\mathrm{AC}$ drives, dynamic analysis of the electrical machines, intelligent control techniques and advanced DSP's. 